\title{
INVESTIGATIONS WITH MODE DIVISION MULTIPLEXED TRANSMISSION
}

\author{
Devendra Kr.Tripathi*, Pallavi Singh, N.K.Shukla, H.K.Dixit \\ J.K.Institute of Applied Physics \&Technology, University Of Allahabad, \\ Allahabad, India
}

\begin{abstract}
This article presents proposed topology for mode division multiplexed link with SPDML and VCSEL, modulate electrical signal of $820 \mathrm{~nm}$ wavelength. Transmission performance has been investigated for numerous modes, bit rates (2.5 to 10Gbps), transmission lengths of MMF (1 to 100km) and attenuation factor in conjunction with parabolic operational mode. Results illustrated successful performance up to nine modes in mode-division multiplexed transmission. Performance is improved at low data rates, illustrated higher OSNR with lower BER,-5dB attenuation factor. Though, at higher bit rate and longer transmission length received OSNR is unsteady. It further inferred that transmission with large number of multiplexed modes result in degraded performance, augmented with minimization of mode coupling losses. Mode division multiplexed multimode optical communication can be a viable option for supplementary capacity augment in the preexisting and future fiber optic communication links.
\end{abstract}

Key words- Differential mode group delay (DMGD), spatial compressed optical receiver (SCOR), spatial direct modulated laser (SDML), weakly coupled-few mode fiber (WCFMF), highly-coupled $(H C F M F)$.

\section{Introduction}

Swift growth in internet traffic, trendy multimedia and smart phone uses are persistently growing so the requested amount of data capacity has been increased pushing-up the capacity demand of preexisting communication networks. So, to keep up with rapid growth of the data capacity lots of novel scheme together with most advanced employed components are being explored persistently to further enhance the transmission performance of Fiber Optic Communication (FOC) systems [1].In the FOC systems optical fiber play significant part and virtually enormous bandwidth offered by optical fibers caused great increase in data transmission speed hence stimulated high-demand multimedia services. Moreover conventionally single mode fiber (SMF) and multimode fibers (MMF) are frequently adopted with optical fiber communication due to their respective pros and cons. However, single-mode fiber (single core) FOC networks transmission capacity is finding to its maximum performance limit.

So, fiber optical communication world will view capacity crisis thus innovative schemes have to be developed for capacity augment. For that, number of multiplexing techniques has been explored from time to time with their respective pros and cons.Amonst many of the multiplexing schemes mode-division multiplexing (MDM) is one of the swiftly emerging scheme. It is getting

DOI : 10.14810/elelij.2014.3304 
more concentration due to maturity of digital signal processing, in a different way with growing capacity need over signals per fiber core. Lot of signals can be transmitted through propagation modes, on any particular core and particular wavelength. For implementation of modes multiplexers and demultiplexer, optical devices are used [2-4].Though mode-division multiplexed transmission systems performance reported remained ambiguous.MDM investigation can be segmented as weakly coupled- few mode fiber (WCFMF) and highly-coupled (HCFMF). HCFMF needs optical system producing very limited mode coupling, in between the modes cross-talk may occur due to lack of cross talk compensation, thus transmission length is affected. While WCFMF is with very less differential mode group delay (DMGD), maximum transmission distance decided by highest value of DMGD, liable for delay which has to fit into filter length of MIMO equalizer.

Primary investigation in MDM transmission technique were employed merely on two or three modes as $\mathrm{LP}_{01}$, two degenerate $\mathrm{LP}_{11}$ modes for communication lengths of $4.5 \mathrm{~km} \mathrm{[5],} \mathrm{10km} \mathrm{[6],}$ $26 \mathrm{~km}$ [7], 33km [8], and 40km [9] adequately with little power penalty. Some researchers has also investigated about MDM-WDM transmission with $\mathrm{LP}_{01}$ and two degenerate $\mathrm{LP}_{11}$ modes, with $6 \times 6$ time-domain MIMO equalizer, little mode fiber (FMF), few mode (FM)-EDFA were used transmitted up to 50km [10].Further expansion of MDM transmission with more number of modes needs scalability of digital signal processing(DSP). To combat polarization diversity single mode coherent receiver employs $2 \times 2$ MIMO algorithms. A receiver must have capability to detect modes which fiber supports for MDM transmission and better performance processing achieved with MIMO algorithm. With optical multiple-input multiple-output (MIMO) communication using HOMs, each signal channel be recovered by digital signal processing (DSP) at the receivers in spite of inter-modal crosstalk in the fiber, novel approach which utilizes the higher-order mode (HOM) in multi-mode fiber (MMF) to realize higher transmission capacity has been explored[1114]. However, lots of tremendous work with multimode fiber optic communication has been explored in past. Though, the above reported papers have certain limitations as maximum transmission length, number of transmitted modes, dispersion. These can be further overcome with implementation of novel design with implementation of advanced lasers, optimizing attenuation factor, OSNR. Even though it is uncertain where about the threshold, accordingly investigations were incessantly going on to optimize performance of mode multiplexed optical communication networks with certain constraints and employing parameter modifications.

In this vision, topology has been proposed for mode division multiplexed transmission designed with spatial direct modulated laser (SDML) and with vertical-cavity surface-emitting laser (VCSEL),performance were investigated for numerous significant transmission parameters such as number of modes, bit rate, attenuation factor,OSNR and transmission length. Detailed analysis of the proposed optical mode division multiplexed link, conclusion and discussion of the results are illustrated in the subsequent sections.

\section{The Simulation design presentation}

The designed topology consists of PRBS with pattern length of seven which generates random signal, followed by electrical signal generator with pattern length of seven with maximum and minimum value of the output electrical signal as $10 \mathrm{e}-3$ volts. This electrical output passed to spatial direct modulated laser (SDML) and with vertical-cavity surface-emitting laser (VCSEL) along with the wavelength of $820 \mathrm{~nm}$ separately. 


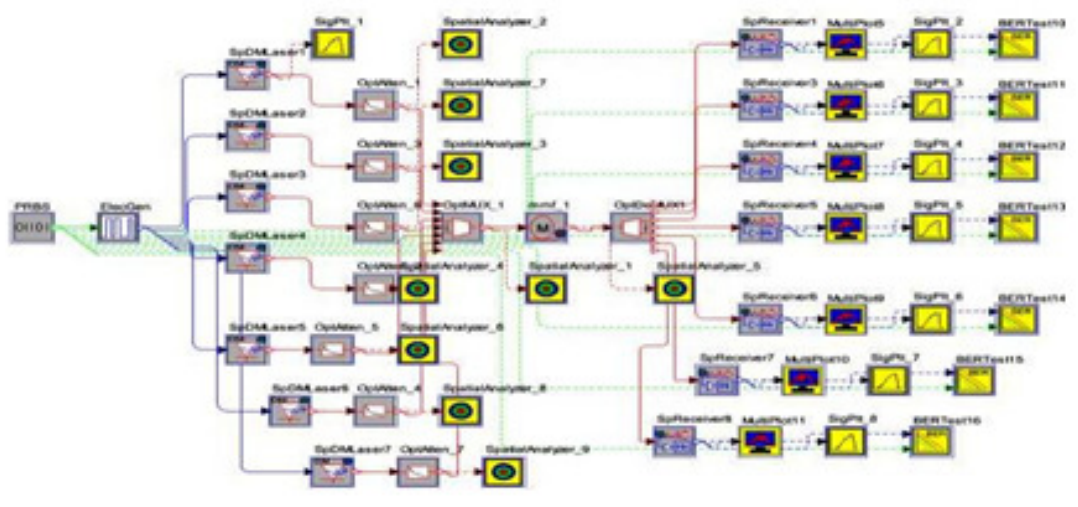

Fig.1 Topology for MDM transmission

This modulated output passed with attenuator which attenuates input signal by the specified level and pass only 20 percent of the power. Attenuated output is further multiplexed in the multiband mode which concatenates the seven independent signals into a linked list which further passes through multimode fiber (MMF). On the receiving end is joined with demultiplexer which splits the fiber output signal. The demultiplexed output signal is passed through spatial compressed optical receiver (SCOR), which is spatial enabled version of the compound optical receiver in which noise is added directly as stochastic contribution to the electrical signal. Low pass Bessel filter chosen with filter bandwidth of 1.0e10 and filter of the fourth order. Analyzing and plotting tools were used for measurement and spatial analyzer is used which is a general purpose measurement tool that produces plots and reports about optical signals with attached spatial fields. In which weighted plot produces superposition of all the detected spatial fields weighted by their time domain values. In this case, the multimode fiber model will produce a linked list of spatio-temporal signals and the multimode fiber model internally multiplexes the time-domain portion of the different sets of signals. For a multimode input having signals of distinct wavelengths, this method cannot be functional since fiber modes and its delay characteristics are dependent upon wavelength.

\section{Results and discussion}

The mode division multiplexed transmission link has been designed and its performance investigated successfully with numerous significant transmission parameters. The performance numerically simulated for bit rates (2.5 to $10 \mathrm{Gbps})$, attenuation factor, number of modes and transmission length ( 1 to $100 \mathrm{~km}$ ). The MDM multimode fiber model generates signal at its output for each of its guided modes and spatial portion of each signal will be the fiber mode itself. The temporal portion of each signal will be a copy of the time-domain part of the input signal modified to reflect the effects of delay, dispersion, attenuation, and coupling. Multimode fiber model generates a linked list of signals, one for each guide fiber mode. Multi mode fiber runs in with parabolic mode and at $820 \mathrm{~nm}$ wavelength analytical fiber model computes numerous distinct guided modes. The cumulative multiplexed SPDML spatial signal is illustrated in the results as shown in different resulting plots. The results are illustrated for plot of the selected input wavelength in the Fig.2 (a) and its magnitude plot in the Fig.2 (b). 

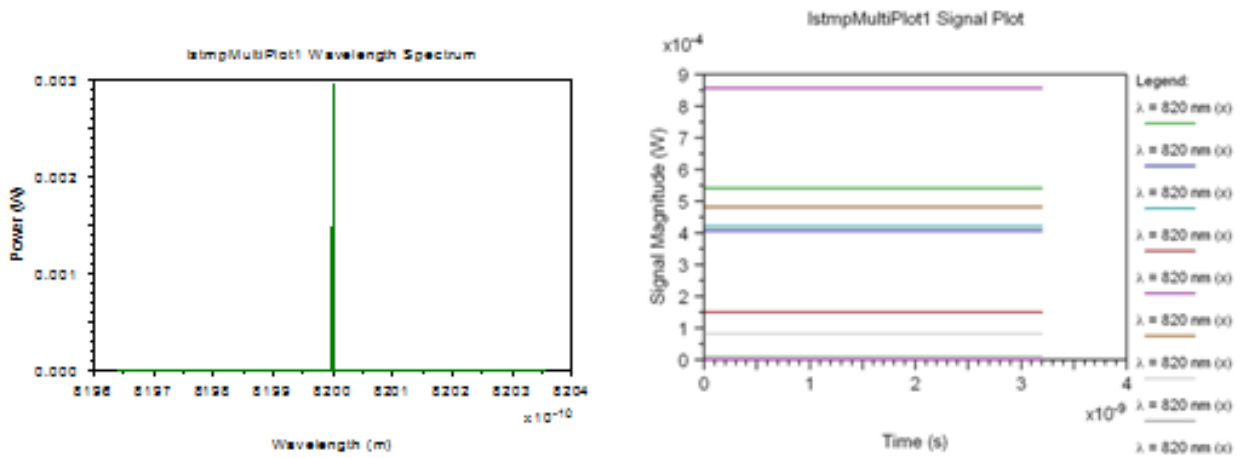

Fig.2 (a) Signal wavelength (b) magnitude plot
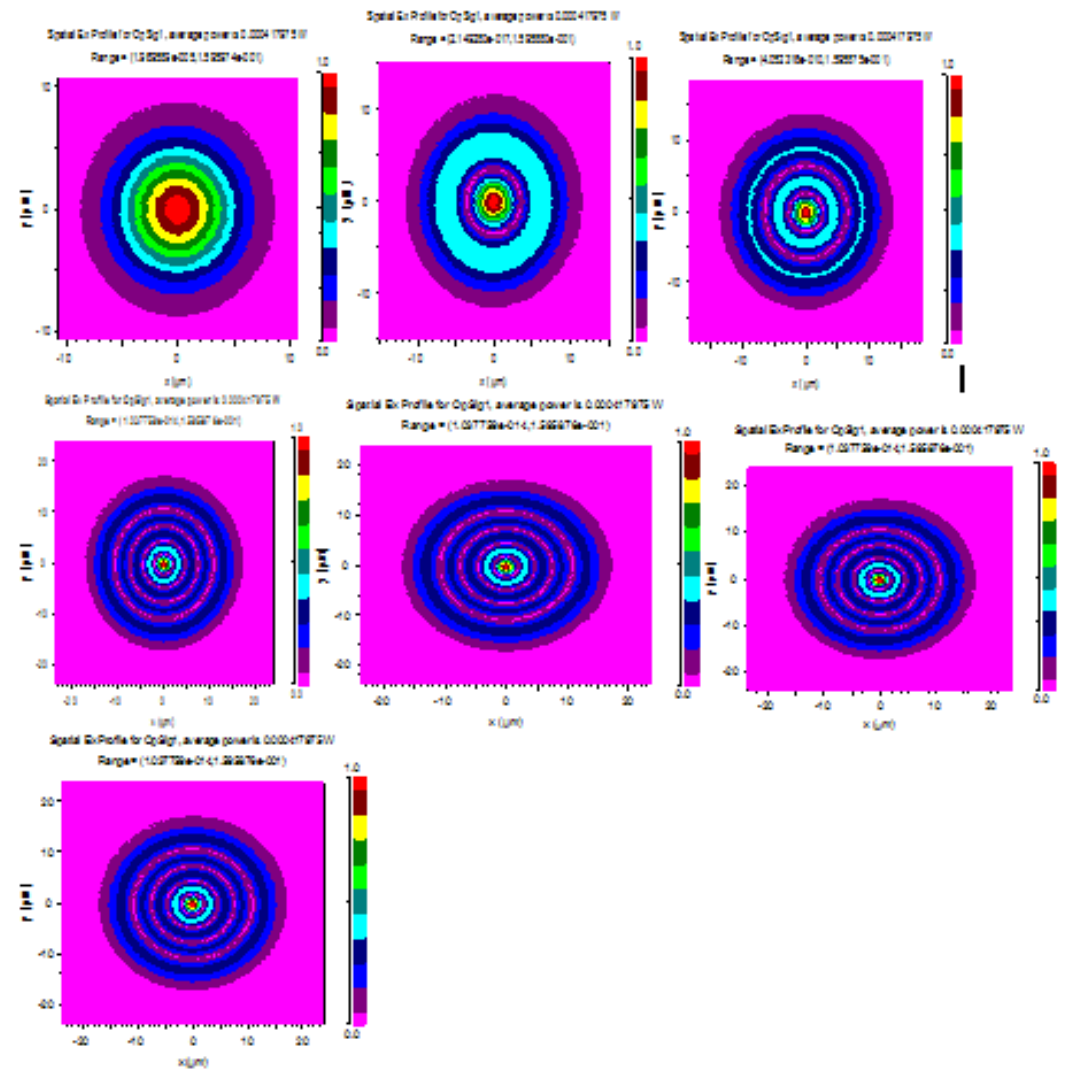

Fig.3 At 10Gbps seven different output mode profiles with each of the SPDML 

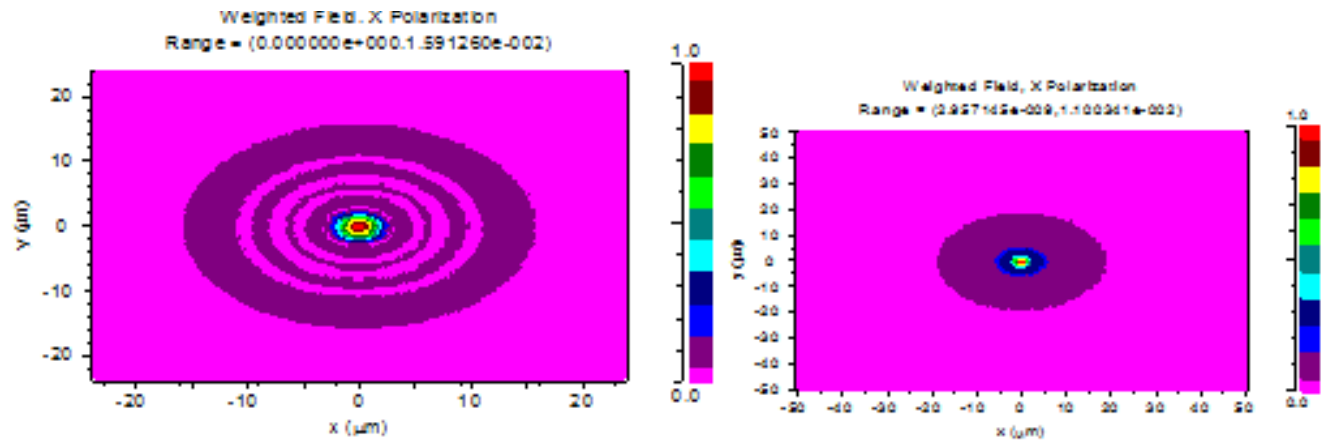

Fig.4 (a) Seven different spatial modes multiplexed together before MMF transmission (b) weighted MDM together after $1 \mathrm{~km}$ MMF and 10Gbps transmission

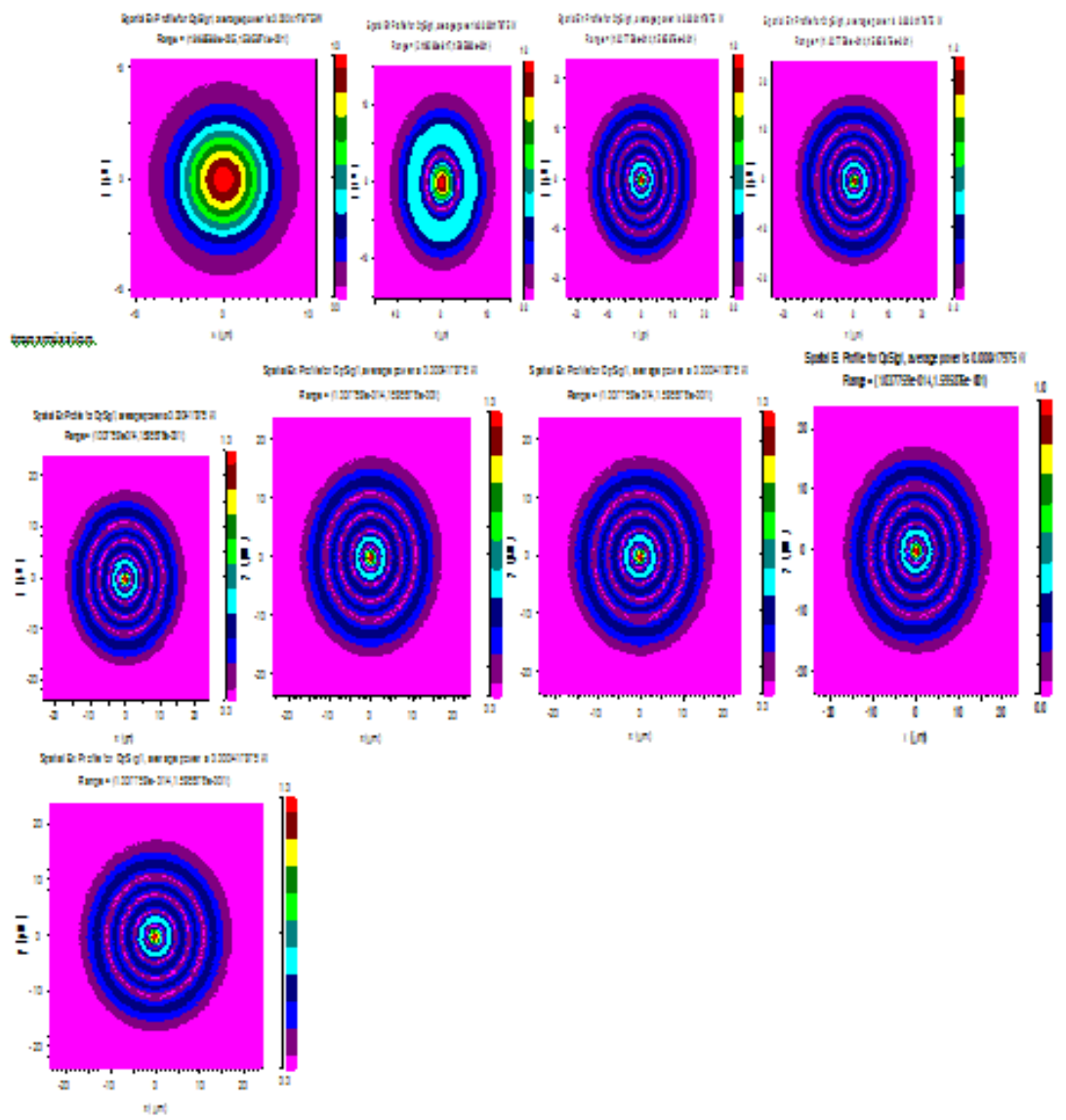

Fig.5 Nine different VCSCEL mode profiles at 10Gbps before transmission 

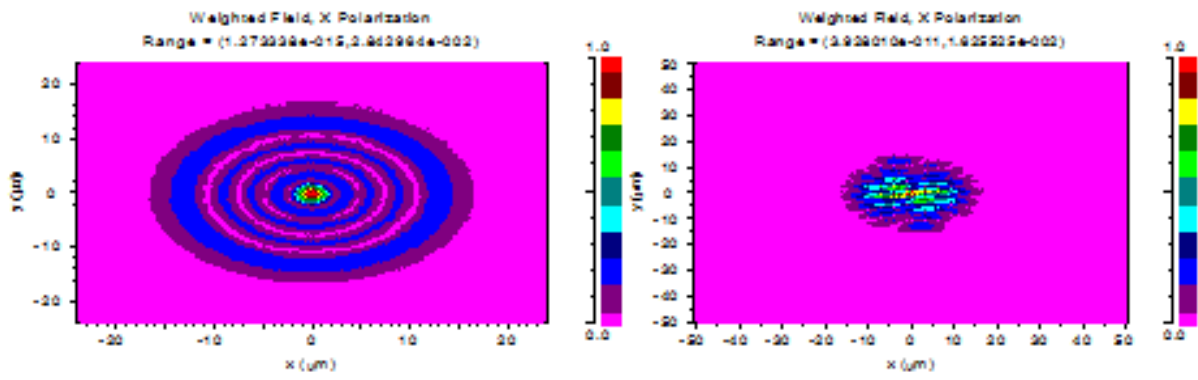

Fig.6 (a) With VCSCEL nine different spatial modes multiplexed together before MMF transmission (b) weighted MDM together after MMF $(50 \mathrm{~km})$ and transmission at $10 \mathrm{Gbps}$ bit rate.

Fig.3 illustrates simulated performance with MDM link at 10Gbps data rate for each of the SPDML spatial mode output profiles, the Fig.4(a) illustrates the SPDML spatial mode multiplexed output profiles before passing through multimode fiber with operating data rates of 10Gbps.Fig.4(b) demonstrates weighted MDM output performance after 1km MMF at 10Gbps bit rate of transmission. Results, inferred that multiplexed mode transmission performance is very good.

Fig.5 illustrates performance with vertical-cavity surface-emitting laser at $10 \mathrm{Gbps}$ data rate for all of the nine output modes before multiplexed. The investigated results with VCSCELs nine different spatial modes multiplexed were illustrated in operating at 10Gbps.Fig.6a illustrates performance with VCSCEL for nine different spatial modes multiplexed before passing through MMF.Fig.6b illustrates resulting transmission performance at MMF (50km) at 10Gbps bit rate with weighted multiplexed (MDM), it inferred that spatial modes on the receiving end show degraded pattern with increase in data rate and number of transmission modes owing to modal dispersion.

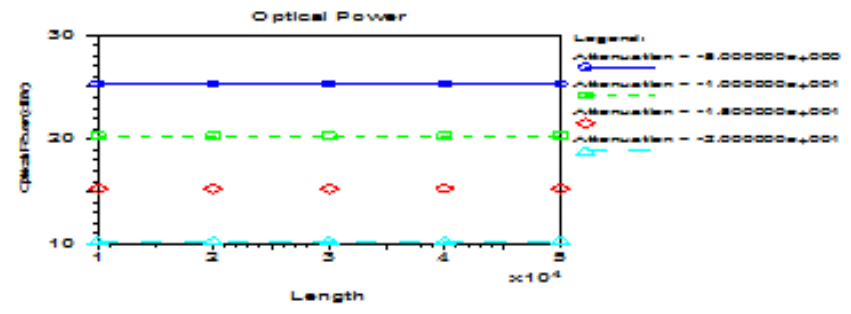

Fig.7 Optical power vs. transmission length against attenuation factor

Fig.7 illustrates transmission performance of MDM link for OSNR against transmission length vs. attenuation, illustrates that higher optical power is achieved with higher values of the attenuation factor so selected attenuation factor should be selected more than $-5 \mathrm{~dB}$ for better transmission performance. 


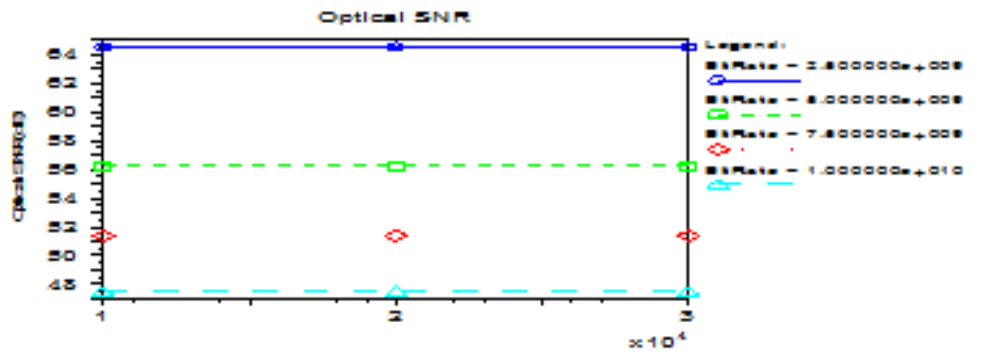

Fig.8 OSNR vs. transmission length against Bit rate

Fig.8 illustrates the investigated results for OSNR vs. transmission length $(30 \mathrm{~km})$ vs. bit rates (2.5 to 10Gbps) for the MDM link. It inferred that received OSNR deteriorates with increase in bit rate, owing to modal dispersion. Though on the lower bit rate transmission show improved transmission performance as modal dispersion is negligible.

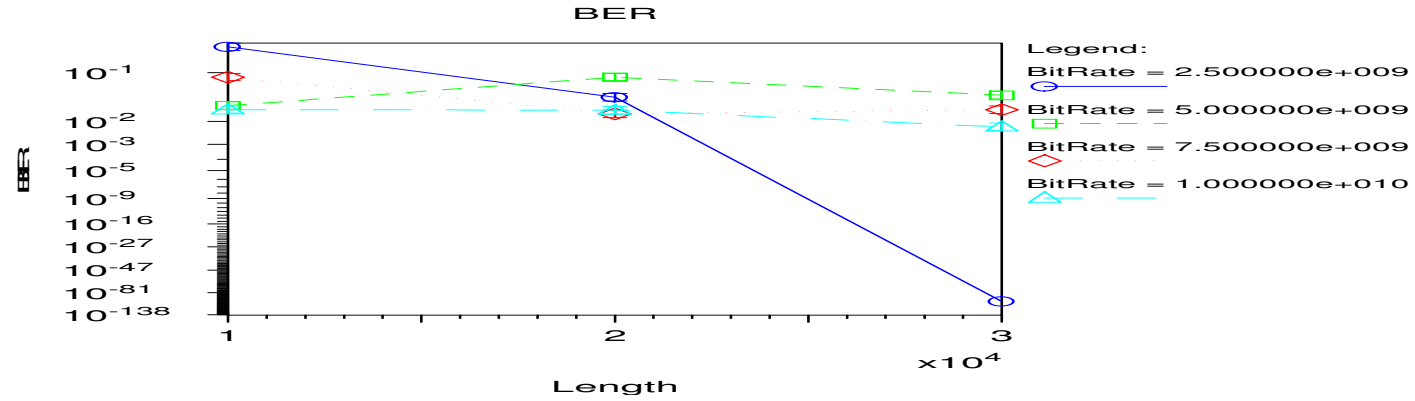

Fig.9 BER vs. transmission length against Bit rate

Fig.9 illustrates MDM links BER transmission performance vs. Bit rate over transmission reach of $30 \mathrm{~km}$, inferred that at higher bit rates BER performance degrades owing to modal dispersions however showed improved performance at lower data rates.
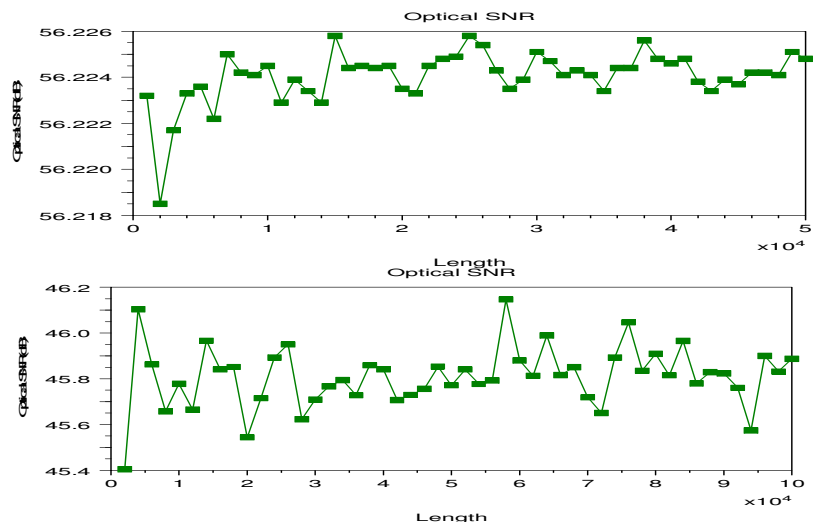

Fig.10 Receiving end OSNR vs. transmission length at $10 \mathrm{Gbps}$ (a) $50 \mathrm{~km}$ (b) $100 \mathrm{~km}$

Fig.10 shows the investigated results for OSNR against transmission length $(50 \mathrm{~km} / 100 \mathrm{~km})$ at bit rate (10Gbps) for mode division multiplexed transmission link. It inferred good OSNR performance though it has unsteady nature to owing to modal dispersions. Moreover the transmission performance investigated illustrate that augment in transmission capacity could be 
achieved with mode division multiplexing joined with higher channel data rates facilitated by the development of electronic and optoelectronic elements in the similar way as wavelength division multiplexing joined with faster ETDM. MDM creates new way for efficient fiber capacity in contrast to solutions based on development of electronic components only as well as tremendous viable option for next generation high-capacity multimode multiplexed fiber optic communication systems.

\section{Conclusion}

To accomplish growing need for data transport mode division multiplexing with MMF link were successfully investigated for mode multiplexing/demultiplexing up to nine modes with modedivision multiplexed transmission. Performance explored for significant parameters to optimize its transmission performance up to $100 \mathrm{~km}$ transmission reach together with SPDML, VCSEL designed MDM topology. Generated signals grouped with optical multiplexer in the multiband mode, joins independent data signals into linked list. It exhibited application of the multiplexer scheme to produce multimode signals while fiber is running in with parabolic operational mode with successful transmission of different modes in mode-division multiplexed transmission Results inferred degraded performance with higher number modes as spatial modes gets degraded, though with minimization of mode coupling losses improved performance achieved altogether with higher OSNR and low BER achieved at lower data rate, $-5 \mathrm{~dB}$ attenuation factor. This study exhibits pathway to enhanced capacity MMF communication. Thus bring into being supplementary aid to the costly preexisting optical transmission networks.

\section{Acknowledgement}

Thanks to J.K.Institute Allahabad (University of Allahabad) for providing the software OptSim(R-Soft) optical communication system.

\section{References}

[1] Z. Xu, Y.J. Wen, W.D. Zhong, T.H. Cheng, M. Attygalle, X. Cheng, Y.K. Yeo, Y.Wang, C. Lu, Characteristics of subcarrier modulation and its application in WDM-PONs, J. Lightw. Technol. (2009)27, 2069-2076.

[2] S. Berdague and P. Facq.Mode division multiplexing in optical fibers. Applied Optics(1982)21, Issue 11:1950-1955.

[3] K. Y. Song et al. High performance fused-type mode selective coupler for two-mode fiber devices.OFC2000:TuB5-2(2000).

[4] F. Saitoh, et al A design method of a fiber-based mode multi/demultiplexer for mode-division multiplexing.Opt. Express (2010)18:4709-4716.

[5] A. Li et al.Reception of mode and polarization multiplexed 107Gb/s co-OFDM signal over a twomode fiber.OFC'11: PDPB8.(2011).

[6] N. Hanzawa et al. Demonstration of mode-division multiplexing transmission over $10 \mathrm{~km}$ two-mode fiber with mode coupler. OFC'11: OWA4 (2011).

[7] A. Al Amin et al.Dual-LP11 mode 4x4 MIMO-OFDM transmission over a two-mode fiber. Opt. Express(2011)19:16672-16679.

[8] S. Randel et al. $6 \times 56-\mathrm{Gb} / \mathrm{s}$ mode-division multiplexed transmission over 33-km few-mode fiber enabled by 6×6 MIMO equalization. Opt. Express(2011)19:16697-16707.

[9] M. Salsi et al. Transmission at $2 \times 100 \mathrm{~Gb} / \mathrm{s}$, over two Modes of 40km-long prototype few mode fiber, using LCOS-based Mode Multiplexer and demultiplexer.OFC'2011: PDPB10 (2011).

[10] Neng Bai, Ezra Ip, Yue-Kai Huang, Eduardo Mateo, Fatih Yaman, Ming-Jun Li,Scott Bickham, Sergey et.al.Mode-division multiplexed transmission with inline few-mode fiber amplifier. OPTICS EXPRESS (30 January 2012)20, No. 3: 2668-2680. 
Electrical and Electronics Engineering: An International Journal (ELELIJ) Vol 3, No 3, August 2014

[11] A.R. Shah, R.C.J. Hsu, A. Tarighat, Coherent optical MIMO (COMIMO), J. Lightw.Technol. (2005) 23. 2410-2419.

[12] A. Tarighat, R.C.J. Hsu, A. Shah, A.H. Sayed, B. Jalali, Fundamentals and challenges of optical multiple-input multiple-output multimode fiber links,IEEE Commun. Mag. (2007)45 (5) 57-63.

[13] B. Franz, D. Suikat, R. Dischler, F. Buchali, H. Buelow, High speed OFDM data transmission over 5 $\mathrm{km}$ GI-multimode fiber using spatial multiplexing with 2 _ 4 MIMO processing, in: Proc. 37th European Conference and Exhibition on Optical Communication (ECOC), paper Th.3.C.4(2011,).

[14] P. Winzer and G. J. Foschini .Outage calculations for spatially multiplexed fiber links.OFC'11: OThO5 (2011). 